\title{
Augmented Paper System: A Framework for User's Personalized Workspace
}

\author{
Kavita Bhardwaj, Santanu Chaudhury, Sumantra Dutta Roy \\ Dept of EE, IIT Delhi \\ New Delhi - 110016, INDIA \\ Email: kavitabhardwaj.iitd@gmail.com, schaudhury@gmail.com, sumantra@ee.iitd.ac.in
}

\begin{abstract}
In this paper, we are presenting a framework for "User's Personalized Workspace" by augmenting the physical paper and digital document. The paper based interactions are seamlessly integrated with digital document based interactions for reading as a activity. For instance when user is involved in reading activity, writing becomes complimentary. In a academic system, paper based presentation mode has facilitated such exercises. Despite rendering the annotation on digital document and store it onto the database, the content of the paper encircled or underlined is used to hyperlink the document. Synchronizing a physical paper and those of digital version in seamless fashion from a user's perspective is the main objective of this work. We have also compared the existing systems which focus on one activity or the other in our proposed system.
\end{abstract}

\section{Keywords:}

Personalized workspace, Augmented paper, Hyperlinking, Annotation rendering

\section{INTRODUCTION}

In [1] The Myth of the Paperless Office authors observed that despite the popularity of computers (desktop or tablets) paper has not lost its advantage of the writing process. For example, paper documents are easy to search as well as easy to annotate while reading and still accepted in many social contexts e.g. in learning, official meetings etc.

A vast research in this field has endured that the activities which can be performed with physical papers can be easily performed with digital documents e.g. drawing annotation, hyperlinking etc.

Some systems only focus on augmenting annotations with digital documents made on physical paper and presents the system for active reading task. Capturing handwritten annotations on a printed document and augmenting them with a digital version of the document has been one of the most frequent issue in pen-and-paper computing. PADD [2] paper augmented digital document system has introduced the main principle of paper-based annotation. This system uses a Anoto digital pen comes with a plug-in for Adobe Acrobat to annotate PDF document. Proofrite [3] is paper-augmented system used to annotate word documents and applies the PADD concept. These annotations are synchronized with digitally stored word documents. The pen-and-paper based interaction is also used for creating and following hyperlinks from printed documents to web pages and the pens used in these systems are ultrasonic pens. PaperLink [4] is one of the system of this class, use a specific kind of pen on which a camera is attached. This camera serves for creating and detecting hot spots and these hots spots are the visual content of the document which serve as link anchors. The Interactive Multimedia Textbook [5] and PapierCraft [6] is also similar example of the same. There are various systems which are developed for one feature or the other. For this work, we collaborate such features with novel techniques and present a system sufficient for a user in academic system. Consider a scenario, a user in academic system deals with lots of paper-based information. When user is involved in reading, writing becomes a complimentary process. These writings are in fact annotations which is considered as a part of reading. We are here proposing this workspace of a augmented paper based reading system to meet these requirements.

The different primitives has been developed for familiar mode of presentation of physical paper and digital document augmentation for reading as a activity. The objective of proposing User's Personalized Workspace is to permit the user for following activities:

- $\quad$ annotate a hard copy of paper while reading and same is rendered on digital document

- $\quad$ as a part of reading, the user may have to find difficult to understand words, the guided usage of the language displays the meaning of such words on desktop by just pointing the word

- during reading by underlining or encircling some text, these annotations are used for content based hyperlinking the document to other related documents.

Steimle et al. [7] designed an integrated framework for various activities but that was specifically proposed for a team work. Compared to this system, our proposed framework is particularly developed for a user's activity in academic system. In our proposed work, we contribute a novel paper based activities augmented with digital document based activities in a seamless integration of both media. In section IV, we discuss a novel annotation technique and the hyperlinking of a paper to its related documents using annotations is described in section $\mathrm{V}$.

Section III explains how the system helps in learning difficult to understand words on paper.

Finally in section VI we conclude the interaction design for our proposed framework "User's Personalized Workspace". 


\section{A FrAMEWORK FOR USER'S PERSONALIZED WORKSPACE}

The grounds for this research is to create a perception driven collaborative workspace which can be used for active reading, learning and referencing to web-pages for a user working on his Desktop. These primitives are synchronized to develop a complete learning environment. The problem focused on incorporating the handwriting (drawing annotations) as a temporal media can be synchronously observed on digital version of the document. The next part is aimed at presenting a realistic augmented learning environment using paper and its digital version. In which a user can learn a language by just pointing the word and the meaning of the word are displayed on dialogue box and in last, on the paper by just encircling and underlining a word the paper can be linked to different documents lying on other portals.

So the overall proposed system has following features:

- Handwriting (drawing annotation) data as a temporal media

- Learning difficult words of a language by pointing the words on the paper with a pen

- Hyperlinking by encircling or underlining the words on the paper with other documents lying on different portals

\section{A. Interactions between Entities}

Figure 1 shows the user interface and the functionality of the system at the back-end. The user's desktop is attached with a digital Inkpad on which physical paper is clipped. User can draw annotations on the digital document and store that annotated document, can select the word for hyperlinking the document and can refer the meaning of difficult to understand words.

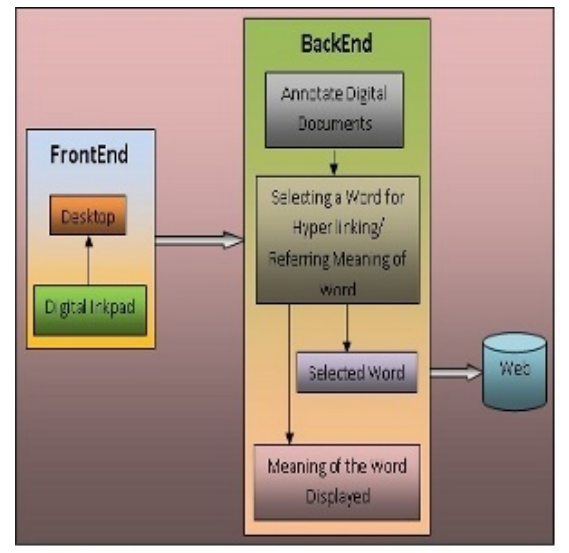

Fig. 1. Interactions occur between entities

\section{Guided UsAGE OF A LANGUAGE}

This application of interactive augmented digital document aimed to help a user by providing meanings of difficult words in his native language, while reading or working on a physical paper. Figure 2 shows the setup used for augmented paper system specifically used for this application.
Figure 2 shows the setup used for the system.

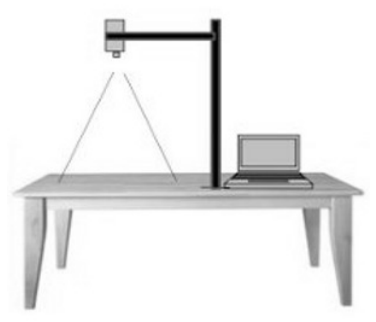

Fig. 2. Setup for augmented paper Framework

This application allows the users to access words on a paper document with a pen gesture based command. The hardware used for this setup are camera, projector and paper computer coordinator. Towards the goal of fine-grained interaction with paper documents, we used content-based physical-digital interaction mapping. The physical-digital interaction mapping basically translates a pen-pointing action on a paper document into an equivalent digital counterpart.

\section{A. Methodology used}

For this SIFT algorithm is used as a content based approach. Scale Invariant Feature Transform(SIFT, hereafter) is an approach for detecting and extracting local feature descriptors that are reasonably invariant to changes in illumination, image noise, occlusion(partially), rotation, scaling, and small changes in viewpoint especially geometric distortions. The scale-invariant feature transform, or SIFT algorithm is among the most well-known and widely-used invariant local feature methods. This method is used for precise coordinate matching between the physical paper and the corresponding digital document by constructing a coordinate transform for the camera and document frames referred. This approach does not require any special markers or modification on physical paper. A SIFT keypoint is a circular image region with an orientation. This is described by a geometric frame of four parameters: the keypoint center $x-y$ coordinates, its scale (the radius of the region), and its orientation. The SIFT detector uses keypoints as image structures which resembles "blobs" and as it is scale invariant it can search the blobs at multiple scales and positions. The keypoints are searched at multiple scales which is obtained by constructing a Gaussian scale space. The scale space is just a collection of images obtained by progressively smoothing the input image, which is analogous to gradually reducing the image resolution. As the SIFT features are obtained between the captured image of the paper and the stored reference image, these are matched to get the initial correspondence. For finding the correspondences circular normalized cross-correlation coefficient is used and the aim is to measure the local intensity similarity between each corner in both images. This circular cross correlation method does not guarantee that all the correspondences are correct, thus introducing outliers. These outliers can severely disturb the estimated transformation and are required to be identified.

A RANSAC algorithm is used to handle this problem by introducing a classification of the data into inliers (valid points) and outliers while estimating the optimal transformation for 
the inliers. A threshold $t$ is used, which ensures that none of the inliers deviates from the model by more than $t$. A small number of data points are selected randomly among the correspondence points and they should satisfy the least square approximation criteria. A transformation model is generated using the least-squares approximation method. All the other correspondence points are transformed with the generated transformation model and classified into inliers and outliers using the threshold $t$. This process is repeated for a specific number of times and the model is re-estimated using the inliers.

The camera captures and analyzes the frames to recognize a portion of the document to detect and trace the user's pen tip. Once the image features of captured image and the preregistered stored image are matched, the correspondences thus formed are used to compute the desired transform between the physical paper and digital document. Now this requires the physical paper to act like a touch screen when accessed with the pen tip. A Meta data file is associated with the user's work in his database at the backend and referred for obtaining the meaning of the word selected by the user. Instead of including information about all the words in the document, the size of the file can be maintained by allowing only selected typical words which are relatively unfamiliar for a language learner. For detecting the pen-tip, there are various approaches but we have used the colour segmentation technique. For our implementation, we have chosen light blue colour for the colour of pen tip, assuming that the pen tip colour is distinguishable from the background. The implementation is done in $\mathrm{C}$ with OpenCV library and a hue based colour segmentation scheme is used for pen tip detection.

Figure 3 shows the images of color segmentation for pen tip detection.

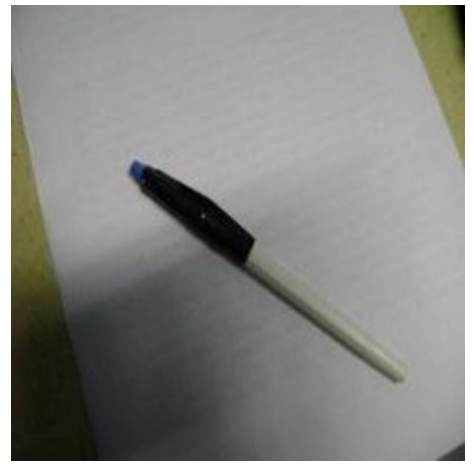

(a) Original image

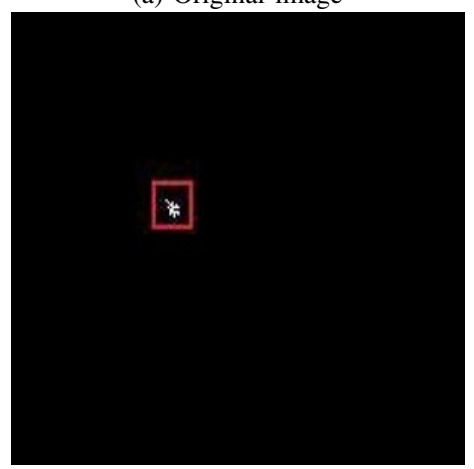

(b) Color segmented binary image

Fig. 3. Colour segmentation method for pen tip detection
The result of projecting meanings of the words on the user's desk is shown in Figure 4.

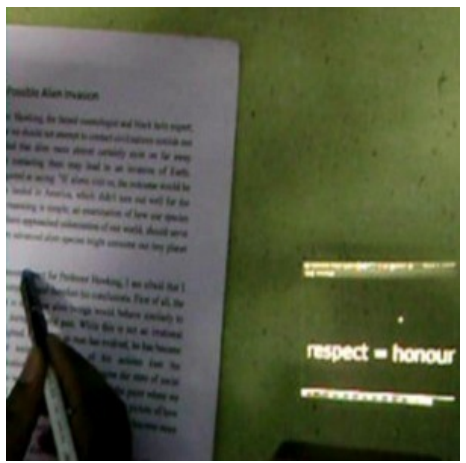

Fig. 4. Meaning of the word projected on user's desk

\section{PERsonalized AnNotation}

User can make handwritten annotation like underlining something important, make synopsis of the read document on physical paper while reading and can use it for future referral. A digital Inkpad is used for making annotations. The user can annotate the physical paper clipped on the Inkpad and these annotations are drawn synchronously on its digital version and can be seen on computer. Figure 5 depicts how annotations rendered on digital document.

\section{A. Capturing Handwritten (drawing annotations) with Tempo- ral Information}

This problem is divided in two parts, first is generation of the annotations on a document and second is rendering of the annotated data on the digitally stored document along with temporal information. To facilitate the use of temporal handwritten data, also termed as ink data, an InkML i.e. Ink Markup Language is used. It supports a complete and accurate representation using time-stamps. The media stream for ink data includes 3 channels $x-y$ coordinate of each pixel of the stroke and $\mathrm{T}$ the time-stamp (Time information e.g. when the annotations made). Each stroke of the handwritten data is denoted as a trace and identified by a unique trace identifier. The time offset contains the time-stamp when rendering of this trace is to be started. Apart from the InkML data we have one more table which includes the set of valid points along with the pixel value. This table is combined with the InkML to generate a set of 4-tuples. Each 4-tuple consists of $\mathrm{x}-\mathrm{y}$ coordinate, time-stamp, pixel value. This set is mapped to the corresponding digital document on which annotation was made.

\section{B. Mapping of Ink data}

Mapping of inkdata onto the digital document is a two step process. 1) mapping of pixel data having $x-y$ coordinate and the RGB value to the temporal data which has $x-y$ coordinate of the pen locations and the time stamp. This mapping is obtained by a shift matrix between temporal and pixel data. Shift matrix is calculated using 4 point correspondence and best fit approximation. Then the time-stamp value was calculated using an elation approach where the pen locations ( $\mathrm{x}-\mathrm{y}$ coord) are elated and the handwritten data is mapped to them. After 
this mapping, we have a data which contains $x-y$ coordinate, time-stamp and pixel value. 2) the data obtained from the prior is $\mathrm{x}-\mathrm{y}$ coordinate, time-stamp and pixel location and it is used for mapping onto the digital document. A transformation matrix is calculated with the correspondence between ink data coordinates to the document's coordinates.

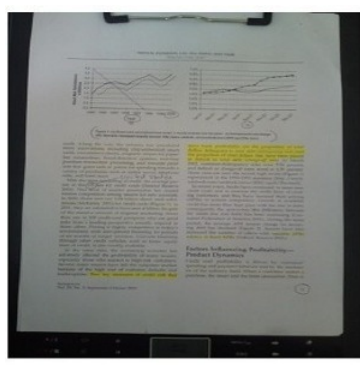

(a) Annotations made on document using Digital Inkpad

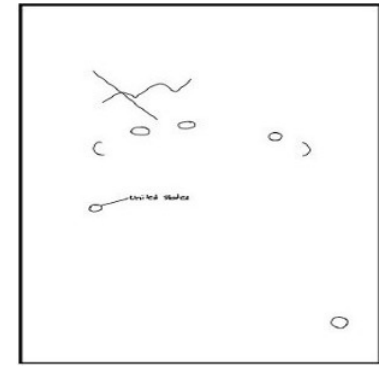

(b) Generated mask of the annotations

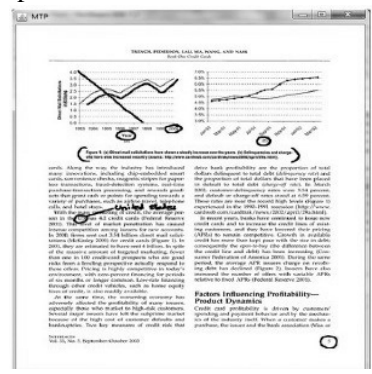

(c) Annotated digital document
Fig. 5. Annotations rendered on digital document

\section{HyPERLINKING THE DOCUMENT}

The annotations made by a user on paper are extracted and inputted to hyperlink module to link the paper with related documents on the web. For reading as a activity, user can select the word on the paper by underlining or encircling (e.g. Keyword of the paper) and the words/text are extracted using OCR and used as a input information to link the paper. The two primary sub modules work in synchronized way to fulfill the task are:

1) marking or highlighting the word on the paper

2) select the highlighted word, recognize using OCR and use it as input to search the related document on the web.

A Digital Inkpad used to highlight a word on the physical paper and the underlined or selected word is extracted from the digital document and used as inputted to the hyperinking. The overall process takes following steps:

1) The document is first divided into horizontal blocks. Each line in the document represents a block.

2) Within each horizontal block the words are separated and the information about each word is stored.

3) Using OCR the selected word is converted into text.

4) When a user writes on a document the number of colored pixel within a word block is counted.

5) If the number is greater than a certain threshold, then the word is marked selected and it is shown on a panel.

\section{A. Hyperlinking of the selected word}

The selected word as described above is linked to a site using ActionListener and Desktop class of java. The selected word is shown on a panel and the user can go to a certain link by clicking on that panel. We have linked the search of the documents for the selected word on the panel with google search engine.

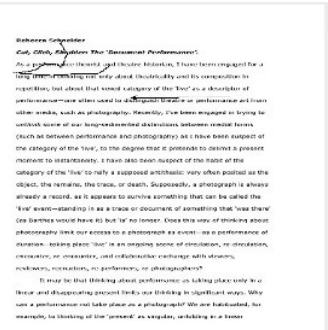

(a) Annotated digital document

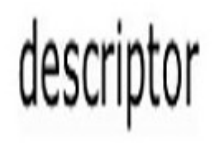

(b) Selected word extracted from the digital document
Fig. 6. Selecting the word for hyperlinking the document

\section{CONCLUSION}

In this paper we handle different problems on augmenting the physical paper and the digital document. The activities that can be performed by the author while reading the physical paper like drawing annotation, further using the annotations for hyperlinking and obtaining the meaning of difficult to understand words etc. can be augmented with digitally stored document. A Digital Inkpad is used for rendering the annotations and selecting the words from the digital document. Integrating these technology and bring it as a Personalized Workspace for a user is the objective of this research.

\section{REFERENCES}

[1] A. J. Sellen and H. Richard, "Harper (2001). the myth of the paperless office."

[2] F. Guimbretière, "Paper augmented digital documents," in Proceedings of the 16th annual ACM symposium on User interface software and technology. ACM, 2003, pp. 51-60.

[3] K. Conroy, D. Levin, and F. Guimbretière, "Proofrite: A paper-augmented word processor," Demo Session of UIST, 2004.

[4] T. Arai, D. Aust, and S. E. Hudson, "Paperlink: a technique for hyperlinking from real paper to electronic content," in Proceedings of the ACM SIGCHI Conference on Human factors in computing systems. ACM, 1997, pp. 327-334.

[5] W.-C. Lai, P.-Y. Chao, and G.-D. Chen, "The interactive multimedia textbook: Using a digital pen to support learning for computer programming," in Advanced Learning Technologies, 2007. ICALT 2007. Seventh IEEE International Conference on. IEEE, 2007, pp. 742-746.

[6] C. Liao, F. Guimbretière, and K. Hinckley, "Papiercraft: a command system for interactive paper," in Proceedings of the 18th annual ACM symposium on User interface software and technology. ACM, 2005, pp. 241-244.

[7] J. Steimle, O. Brdiczka, and M. Muhlhauser, "Coscribe: Integrating paper and digital documents for collaborative knowledge work," Learning Technologies, IEEE Transactions on, vol. 2, no. 3, pp. 174-188, 2009. 